\title{
Hyperkeratosis, CTCAE
}

National Cancer Institute

\section{Source}

National Cancer Institute. Hyperkeratosis, CT CAE. NCI Thesaurus. Code C143246.

A disorder characterized by a thickening of the outer layer of the skin. 